\title{
Tree growth form in the establishment phase of a silvopastoral system in NE Scotland
}

\author{
A.R. Sibbald, V. Clements, V. Campero, A.J.I. Dalziel. \\ MLURI, Craigiebuckler, Aberdeen, AB15 8QH, UK
}

Growth and survival of trees in an upland silvopastoral system have been shown to be positively correlated with planting density. Tree growth form also contributes to the value of the tree crop. In this experiment five silvopastoral treatments, hybrid larch (Larix eurolepis, 100, 200 and 400 trees.ha-1) and sycamore (Acer pseudoplatanus, 100 and 400 trees $^{-h^{-1}}{ }^{-1}$ ) were subjectively assessed for growth form from 0 (dead) to 10 (perfect). Scores 0-6 were classed as 'poor' (for pulping or chipping); scores of 7-10 were classed as 'good' (at least of building quality). The sycamore had previously been form-pruned to produce a single leading shoot. Hybrid larch had previously been pruned to remove branches below $1.8 \mathrm{~m}$ to reduce the sail effect of the canopy. Hybrid larch had $22 \%( \pm 8), 41 \%( \pm 4)$ and $46 \%( \pm 10)$ of 'good' trees at 100,200 and 400 trees ha ${ }^{-1}$ respectively. Sycamore had 55\% $( \pm 12)$ and $85 \%( \pm 5)$ of 'good' trees for 100 and 400 trees.hat $^{-1}$. These results show that good tree growth form is associated with higher planting densities most probably due to animal behaviour effects modifying plant growth conditions (Sibbald et al. [47] (1998) Ann. Zootech. this volume). Trees should not be planted at less than $400 \cdot \mathrm{ha}^{-1}$. Growth form of sycamore was less influenced by the site conditions than that of hybrid larch; hybrid larch had good form in sheltered areas of the site. Tree species should be matched to site conditions.

\section{Les pâturages boisés du Parc Jurassien Vaudois dans le projet intégré GEST- MONTAGNE}

\author{
J.-D. Gallandat ${ }^{\mathrm{a}}$, F. Gillet ${ }^{\mathrm{a}}$, O. Schneider ${ }^{\mathrm{b}}$, M. Brülhmann ${ }^{\mathrm{c}}$, J.-B. Wettstein $^{\mathrm{d}}$
}

"Laboratoire d'écologie végétale de l'Université, 2007 Neuchâtel ; ${ }^{b}$ Bureau BOSFORE, 2035 Corcelles NE ; "Prométerre, 1345 Le Lieu ; "Parc Jurassien Vaudois, 1450 SainteCroix, Suisse

Depuis 1995, le Pare Jurassien Vaudois (CH) est engagé dans la réalisation d'un vaste projet intégré visant une conservation dynamique d'un site de $50 \mathrm{~km}^{2}$. Le maintien de l'économie sylvicole et de l'économie pastorale devrait garantir la conservation de forêts et de pâturages boisés d'une grande valeur biologique. Le projet contribue également à la sauvegarde du patrimoine bâti. Agronomes, forestiers et phytosociologues ont élaboré un plan de gestion intégré qui s'articule autour des quatre axes suivants : i) maintien du taux et de la répartition des boisés grâce à des systèmes d'exploitation prenant en compte la diversité des milieux ; l'équilibre dynamique des ligneux et des herbacées dépend de la maitrise du chargement animal ; ii) correction ou prévention des déséquilibres. Dans le cadre du programme de recherches PATUBOIS, l'Université de Neuchâtel a mis au point des outils intégrés de diagnostic et de simulation dynamique à partir de l'observation fine du couvert végétal ; iii) conservation à long terme des richesses naturelles et du patrimoine bâti afin d'ancrer les produits dans le territoire et de favoriser un tourisme adapté aux contraintes de cette conservation ; iv) coordination des mesures nécessaires au maintien des pâturages boisés et choix des investissements en fonction des objectifs de conservation et des capacités financières des propriétaires et des exploitants. 